KEK-TH-1322

IPMU-09-0083

arXiv:0907.3832

\title{
Topological String on $\operatorname{OSP}(1 \mid 2) / \mathrm{U}(1)$
}

\author{
Gaston Giribet ${ }^{a *}$, Yasuaki Hikida ${ }^{4}$ and Tadashi Takayanagi $₫$ \\ ${ }^{a}$ Physics Department, University of Buenos Aires, and Conicet, \\ Ciudad Universitaria, Pab. I, 1428, Buenos Aires, Argentina \\ ${ }^{b}$ KEK Theory Group, Tsukuba, Ibaraki 305-0801, Japan \\ ${ }^{c}$ Institute for the Physics and Mathematics of the Universe (IPMU), \\ University of Tokyo, Kashiwa, Chiba 277-8582, Japan
}

\begin{abstract}
We propose an equivalence between topological string on $\operatorname{OSP}(1 \mid 2) / \mathrm{U}(1)$ and $\hat{c} \leq 1$ superstring with $\mathcal{N}=1$ world-sheet supersymmetry. We examine this by employing a free field representation of $\operatorname{OSP}(1 \mid 2)$ WZNW model and find an agreement on the spectrum. We also analyze this proposal at the level of scattering amplitudes by applying a map between correlation functions of OSP(1|2) WZNW model and those of $\mathcal{N}=1$ Liouville theory.
\end{abstract}

\footnotetext{
*E-mail: gaston@df.uba.ar

${ }^{\dagger}$ E-mail: hikida@post.kek.jp

${ }^{\ddagger}$ E-mail: tadashi.takayanagi@ipmu.jp
} 


\section{Contents}

1 Introduction $\quad 1$

$2 \mathcal{N}=2$ Coset $\operatorname{OSP}(1 \mid 2) / \mathrm{U}(1)$ and 2D Superstring 2

$2.1 \mathrm{OSP}(1 \mid 2)$ Current Algebra . . . . . . . . . . . . . . . . 3

$2.2 \mathcal{N}=2$ Supersymmetric Coset Model . . . . . . . . . . . . 5

2.3 Topological Twisting . . . . . . . . . . . . . . . . 6

2.4 Chiral Primaries . . . . . . . . . . . . . . . . . 7

$3 \operatorname{OSP}(1 \mid 2) / \mathrm{U}(1)$ Coset from $\mathcal{N}=1$ Super Liouville

$3.1 \operatorname{OSP}(1 \mid 2)$ WZNW Model . . . . . . . . . . . . . . . . . . . 9

$3.2 \mathrm{OSP}(1 \mid 2)-$ Super Liouville Correspondence . . . . . . . . . . . . . . . . 11

3.3 Amplitudes of $\operatorname{OSP}(1 \mid 2) / \mathrm{U}(1)$ Coset Model . . . . . . . . . . . . . . . . 12

4 Correspondence to $\hat{c} \leq 1$ Superstring Theory $\quad 14$

$4.1 \hat{c} \leq 1$ Superstring Theory . . . . . . . . . . . . . . . . . . . . 14

4.2 Amplitudes of Topological Model . . . . . . . . . . . . . . . . 15

4.3 Comparison of Correlation Functions . . . . . . . . . . . . . . . . . 16

5 Conclusion and Discussions $\quad 17$

\begin{tabular}{ll|} 
A Free Field Correlation Functions & 18 \\
\hline
\end{tabular}

\section{Introduction}

Superstrings on AdS spaces have been widely studied by virtue of their applications to the holographic duality, i.e. the AdS/CFT correspondence [1]; and it has become clear that the structure of supergroup $\sigma$-models is of great importance to investigate superstrings in these spaces. For instance, the supergroup $\operatorname{PSU}(2,2 \mid 4)$ turns out to be important to construct superstring theory on $A d S_{5} \times S^{5}$ [2]. Besides, superstring theory on $A d S_{3} \times S^{3}$ can be described in terms of the PSL(1,1|2) WZNW model [3]. However, in spite of its importance, quantizing supergroup $\sigma$-models is a quite difficult problem, and hence solving superstring theory on AdS spaces exactly still remains as an unsolved question.

Fortunately, there is a simpler type of duality for which string world-sheet theory is still described by a supergroup WZNW model. It has been established in [4, 5] that two-dimensional superstring (type 0 string) can be holographically described by a simple Hermitian matrix model. At present, this is the only dynamical model of string theory which is non-perturbatively well-defined and is exactly solvable even at finite temperature. The two-dimensional type 0 string theory is originally defined by string world-sheet theory 
with the $\hat{c}=1$ matter coupled to $\mathcal{N}=1$ super Liouville theory. Boosting the linear dilaton with Liouville potential kept the same, this theory can be extended to $\hat{c} \leq 1$ type 0 string theory as it has been done for bosonic string in [6, 7]. Note that dual matrix model can be constructed even for $\hat{c}<1$ case, as shown in [6].

In this paper, we argue that these $\hat{c} \leq 1$ superstring theories can be described by utilizing the supergroup $\operatorname{OSP}(1 \mid 2) 1$ Precisely speaking, we propose that the $\hat{c} \leq 1$ superstring is equivalent to topological string on $\mathcal{N}=2$ superconformal coset $\operatorname{OSP}(1 \mid 2) / \mathrm{U}(1) 2$ This relation can be thought of as a supersymmetric version of the known relation between $c \leq 1$ bosonic string theory and topological string on $\mathrm{SL}(2) / \mathrm{U}(1)$ [14, 7]. This extension might be guessed from the quantum Hamiltonian reduction since OSP(1|2) WZNW model is reduced to $\mathcal{N}=1$ super Liouville theory [15], just like $\mathrm{SL}(2)$ WZNW model is reduced to bosonic Liouville theory [16].

This paper is organized as follows. In the next section, we explicitly construct the $\mathcal{N}=2$ superconformal coset $\operatorname{OSP}(1 \mid 2) / \mathrm{U}(1)$ as a natural extension of Kazama-Suzuki model for bosonic cosets [17, 18, 3. We analyze it in the free field theory and show that the $\hat{c} \leq 1$ string world-sheet appears after the topological twisting. In particular, we show that free fields in the coset model become the matter contents of $\hat{c} \leq 1$ superstring, and the chiral primaries of the coset model are identified with the physical operators of $\hat{c} \leq 1$ superstring. In section 3, we review and extend the map between the correlation functions in $\operatorname{OSP}(1 \mid 2)$ WZNW model and those in the $\mathcal{N}=1$ Liouville theory. This relation was originally obtained in [21] as an generalization of $H_{3}^{+}$-Liouville relation [22, 23]. In section 4 , after briefly reviewing $\hat{c} \leq 1$ superstrings, we apply this map to study the scattering S-matrices. We explicitly show that the correlation functions of physical operators in the topological model are mapped to those of physical operators in the $\hat{c} \leq 1$ superstring. In section 5, we summarize the conclusion. In the appendix, we discuss correlation functions of $\operatorname{OSP}(1 \mid 2)$ WZNW model in the free field representation.

\section{$2 \mathcal{N}=2$ Coset $\operatorname{OSP}(1 \mid 2) / \mathrm{U}(1)$ and 2D Superstring}

In this section we construct and analyze $\mathcal{N}=2$ supersymmetric coset (Kazama-Suzuki model [17, 18]) based on $\operatorname{OSP}(1 \mid 2) / \mathrm{U}(1)$. After its topological twisting, we show explicitly from the free field theory analysis that the world-sheet theory of $\hat{c} \leq 1$ superstring indeed appears. We also discuss chiral primary states which are the physical states in the topologically twisted theory.

\footnotetext{
${ }^{1}$ Current superalgebra of OSP type also appears in an attempt [8] to generalize heterotic string so as to be dual to Type I string theory with a OSP gauge symmetry.

${ }^{2}$ Topological strings on cosets based on sugerpgroups have been studied for the analysis of Maldacena conjecture via world-sheet theory in [9, 10, 11, 12, 13.

${ }^{3}$ Generic construction of Kazama-Suzuki model for cosets of supergoups was given in [19, 20, very recently.
} 


\subsection{OSP(1|2) Current Algebra}

The current algebra of $\operatorname{OSP}(1 \mid 2)$ includes SL(2) bosonic subalgebra, which is generated by $J^{3}(z)$ and $J^{ \pm}(0)$ with their OPEA 4

$$
J^{+}(z) J^{-}(0) \sim \frac{k}{z^{2}}-\frac{2 J^{3}(0)}{z}, \quad J^{3}(z) J^{ \pm}(0) \sim \pm \frac{J^{ \pm}(0)}{z}, \quad J^{3}(z) J^{3}(0) \sim-\frac{k}{2 z^{2}} .
$$

In addition to these bosonic generators, there are fermionic ones with

$$
\begin{aligned}
& J^{3}(z) j^{ \pm}(0) \sim \pm \frac{j^{ \pm}(0)}{2 z}, \quad J^{ \pm}(z) j^{\mp}(0) \sim \mp \frac{j^{ \pm}(0)}{z}, \\
& j^{+}(z) j^{-}(0) \sim \frac{2 k}{z^{2}}-\frac{2 J^{3}(0)}{z}, \quad j^{ \pm}(z) j^{ \pm}(0) \sim-\frac{2 J^{ \pm}(0)}{z} .
\end{aligned}
$$

The energy momentum tensor is given by Sugawara construction and the central charge is $c=2 k /(2 k-3)$. These are the definition of $\operatorname{OSP}(1 \mid 2)$ current algebra with level $k$.

In a free field representation [24, 15, 25, 26] the above currents may be expressed as

$$
\begin{aligned}
& J^{-}=\beta, \quad J^{+}=\beta \gamma^{2}-\frac{1}{b} \gamma \partial \phi+\gamma \theta p+k \partial \gamma-(k-1) \theta \partial \theta \\
& J^{3}=\beta \gamma-\frac{1}{2 b} \partial \phi+\frac{1}{2} \theta p, \quad j^{-}=p-\beta \theta, \quad j^{+}=\gamma p-\beta \gamma \theta+\frac{1}{b} \theta \partial \phi-(2 k-1) \partial \theta
\end{aligned}
$$

where the OPEs of these free fields are

$$
\phi(z) \phi(0) \sim-\ln z, \quad \beta(z) \gamma(0) \sim \frac{1}{z}, \quad p(z) \theta(0) \sim \frac{1}{z} .
$$

The field $\phi$ has the background charge $Q_{\phi}=b$ and the central charge is $c=1+3 Q_{\phi}^{2}$. Here the parameter $b$ is related to the level $k$ as $1 / b^{2}=2 k-3$. The bosonic fields $(\beta, \gamma)$ have conformal weights $(1,0)$ and the central charge of this system is $c=2$. On the other hand, $(p, \theta)$ are fermions with conformal weights $(1,0)$ and central charge $c=-2$. In the following analysis, it is useful to bosonize the fermionic fields $(p, \theta)$ as

$$
\theta=e^{i Y}, \quad p=e^{-i Y}
$$

For instance, the $J^{3}$ current takes the form

$$
J^{3}=\beta \partial \gamma-\frac{1}{2 b} \partial \phi+\frac{i}{2} \partial Y
$$

The energy momentum tensor is given by

$$
T=\beta \partial \gamma-\frac{1}{2} \partial \phi \partial \phi+\frac{b}{2} \partial^{2} \phi-p \partial \theta=\beta \partial \gamma-\frac{1}{2} \partial \phi \partial \phi+\frac{b}{2} \partial^{2} \phi-\frac{1}{2} \partial Y \partial Y+\frac{i}{2} \partial^{2} Y
$$

in terms of these free fields.

\footnotetext{
${ }^{4}$ For a while we concentrate on the holomorphic part.
} 
One of the merits to utilize the free field representation is that vertex operators can be expressed in a simple form. Namely, the vertex operators of $\operatorname{OSP}(1 \mid 2)$ model can be written in terms of free fields as

$$
\Phi_{j, m}^{s} \sim e^{i s Y} \gamma^{j-s / 2+m} e^{-2 b j \phi}
$$

whose conformal weight with respect to (2.7) is

$$
\Delta=-2 b^{2} j\left(j+\frac{1}{2}\right)+\frac{1}{2} s(s-1) .
$$

Here we set $s=0,1 / 2,1$. For $s=0,1$ we can express the vertex operator even in terms of $\theta$, but not for $s=1 / 2$. The operator with $s=1 / 2$ corresponds to a twist operator in R-sector, whose role was argued for GL(1|1) WZNW model in [27], see also [28]. In order to compute correlation functions, the overall normalization of vertex operators should be fixed. More precise definition will be given in section 3. Correlation functions of vertices (2.8) are discussed in the appendix, where the Coulomb gas prescription is given.

Notice that the expression (2.9) is invariant under the Weyl transformation $j \rightarrow-j-$ $1 / 2$ and under $s \rightarrow 1-s$. This allows us to consider a second contribution to (2.8) which goes like $\sim e^{2 b(j+1 / 2) \phi}$ and it would dominate the large $\phi$ regime for $j>-1 / 4$. In addition, there exist conjugate representations which are similar to those that exist in the free field realization of SU(2) model [29, 30, 25, 26]. For instance, one finds the operator

$$
\hat{\Phi}_{j, j+\frac{1}{2}}^{0} \sim \beta^{k-2-2 j} e^{b(2 k-3-2 j) \phi}
$$

which represents a Kac-Moody primary of conformal dimension (2.9), with $m=j+1 / 2$ and $s=0$. It can be also thought of as a conjugate representation for the state with $m=j, s=1$.

In order to define the $\operatorname{OSP}(1 \mid 2) / \mathrm{U}(1)$ coset theory, we utilize the representation introduced in [31] and [32] to realize the $S L(2) / U(1)$ coset theory. This amount to introduce a boson $X^{3}(z)$ with $X^{3}(z) X^{3}(0) \sim-\ln z$, as well as a $(b, c)$ ghost system, which are used to mode out the $U(1)$ factor. Then the vertex operators of the coset theory are given by

$$
\Phi_{j, m}^{s}=\Psi_{j, m}^{s} e^{-i \sqrt{\frac{2}{k}} m X^{3}}
$$

As we will discuss below, the $\operatorname{OSP}(1 \mid 2)$ current algebra admits the symmetry under the spectral flow action as in the case of SL(2) WZNW model [33]. For OSP(1|2) model, spectrally flowed states are defined in this form as

$$
\Phi_{j, m}^{s, w}=\Psi_{j, m}^{s} e^{-i \sqrt{\frac{2}{k}}\left(m+\frac{k}{2} w\right) X^{3}}
$$

with the index of spectral flow $w$. 


\section{2 $\mathcal{N}=2$ Supersymmetric Coset Model}

In this subsection we would like to construct $\mathcal{N}=2$ supersymmetric model based on the coset $\operatorname{OSP}(1 \mid 2) / \mathrm{U}(1)$. For the purpose we first generalize the $\operatorname{OSP}(1 \mid 2)$ current algebra into $\mathcal{N}=1$ supersymmetric version, therefore we need superpartners of currents $\left(J^{3}, J^{ \pm}, j^{ \pm}\right)$. While we introduce fermions $\left(\psi^{3}, \psi^{ \pm}\right)$with spin $1 / 2$ for the bosonic currents $\left(J^{3}, J^{ \pm}\right)$, we include bosons $\varphi^{ \pm}$with spin $1 / 2$ for the fermionic currents $j^{ \pm}$. We assume the OPEs of these fields as

$$
\psi^{+}(z) \psi^{-}(0) \sim \frac{1}{z}, \quad \psi^{3}(z) \psi^{3}(0) \sim \frac{1}{z}, \quad \varphi^{+}(z) \varphi^{-}(0) \sim-\frac{1}{z} .
$$

Notice that the extra bosons $\varphi^{ \pm}$satisfy wrong spin-statistic relation. With these new fields we can define $\mathcal{N}=1$ supercurrents as

$$
\hat{J}^{ \pm}=J^{ \pm}+\sqrt{2} \psi^{ \pm} \psi^{3}+\frac{1}{2} \varphi^{ \pm} \varphi^{ \pm}, \quad \hat{j}^{ \pm}=j^{ \pm} \pm i \sqrt{2} \psi^{ \pm} \varphi^{\mp} \pm i \psi^{3} \varphi^{ \pm},
$$

along with

$$
\hat{J}^{3}=J^{3}+\psi^{+} \psi^{-}+\frac{1}{2} \varphi^{+} \varphi^{-} .
$$

We construct the coset model by using the last current as well as removing one of the fermions $\psi^{3}$ to preserve $\mathcal{N}=1$ world-sheet supersymmetry.

We can show that the coset model actually has enhanced $\mathcal{N}=2$ supersymmetry as Kazama-Suzuki models for bosonic cosets [17, 18]. We find that the generators of $\mathcal{N}=2$ superconformal symmetry are

$$
\begin{aligned}
J_{R}= & -\frac{1}{2 k-3}\left(2 J^{3}+(2 k-1) \psi^{+} \psi^{-}+(2 k-2) \varphi^{+} \varphi^{-}\right) \\
G^{ \pm}= & \frac{1}{\sqrt{2 k-3}}\left(2 J^{ \pm} \psi^{\mp} \pm \sqrt{2} j^{ \pm} \varphi^{\mp}+\left(\varphi^{\mp}\right)^{2} \psi^{ \pm}\right), \\
T= & \frac{1}{2 k-3}\left[J^{+} J^{-}+J^{-} J^{+}+\frac{1}{2}\left(j^{-} j^{+}-j^{+} j^{-}\right)+4 J^{3} \psi^{+} \psi^{-}+2 J^{3} \varphi^{+} \varphi^{-}+2 \psi^{+} \psi^{-} \varphi^{+} \varphi^{-}\right. \\
& \left.-\frac{2 k+1}{2}\left(\psi^{+} \partial \psi^{-}+\psi^{-} \partial \psi^{+}\right)-(k-1)\left(\varphi^{+} \partial \varphi^{-}-\varphi^{-} \partial \varphi^{+}\right)+\frac{1}{2}\left(\varphi^{+}\right)^{2}\left(\varphi^{-}\right)^{2}\right] .
\end{aligned}
$$

In fact, we can compute the OPEs of generators as

$$
\begin{aligned}
& T(z) T(0) \sim \frac{c / 2}{z^{4}}+\frac{2 T(0)}{z^{2}}+\frac{\partial T(0)}{z}, \quad T(z) G^{ \pm}(0) \sim \frac{\frac{3}{2} G^{ \pm}(0)}{z^{2}}+\frac{\partial G^{ \pm}(0)}{z}, \\
& T(z) J_{R}(0) \sim \frac{J_{R}(0)}{z^{2}}+\frac{\partial J_{R}(0)}{z}, \quad J_{R}(z) G^{ \pm}(0) \sim \pm \frac{G^{ \pm}(0)}{z}, \quad J_{R}(z) J_{R}(0) \sim \frac{c / 3}{z^{2}}, \\
& G^{ \pm}(z) G^{\mp}(0) \sim \frac{\frac{2}{3} c}{z^{3}} \pm \frac{2 J_{R}(0)}{z^{2}}+\frac{2 T(0)}{z} \pm \frac{\partial J_{R}(0)}{z}, \quad G^{ \pm}(z) G^{ \pm}(0) \sim 0 .
\end{aligned}
$$

In this way we have explicitly shown that these generators satisfy the $\mathcal{N}=2$ superconformal algebra with central charge $\hat{c}=c / 3=1 /(2 k-3)$. 


\subsection{Topological Twisting}

To realize the U(1) quotient more explicitly, we combine a free scalar field $X$ with the $\mathcal{N}=1$ OSP current algebra and finally take a quotient by the complexified U(1) (i.e. the complex plane $\mathbb{C}$ ). This quotient can be done by taking BRST invariant state about the BRST operator

$$
Q_{B}=\int d z C(z) J_{g}(z)
$$

where we introduced fermionic ghosts $(B, C)$ with the conformal weights $(1,0)$. Here the BRST current $J_{g}$ is defined by

$$
J_{g}=\hat{J}^{3}-\frac{i}{2 b} \partial X
$$

and it is easy to see that $J_{g}(z) J_{g}(0) \sim 0$ which guarantees $Q_{B}^{2}=0$.

Notice that in this formalism we can always set $J_{g}(z)$ to zero since it is gauged. Using this fact we can use the following expression of the R-current of $\mathcal{N}=2$ superconformal algebra as

$$
J_{R}^{\prime}=J_{R}-\frac{4 k-8}{2 k-3} J_{g}=-2 J^{3}-3 \psi^{+} \psi^{-}-2 \varphi^{+} \varphi^{-}+i \frac{2 k-4}{\sqrt{2 k-3}} \partial X .
$$

In the anti-holomorphic part, we use the same expression with bars 5 We will find that this form of R-current is useful to construct the topological model as in the bosonic case [14, 7].

Now, we perform topological twists [34, 35] by using the expression (2.20) of R-current. Namely, we redefine the energy momentum tensor by $T^{\text {top }}=T+\frac{1}{2} \partial J_{R}^{\prime}$ and $\bar{T}^{\text {top }}=$ $\bar{T}+\frac{1}{2} \bar{\partial} \bar{J}_{R}^{\prime}$. Employing the free field representation (2.3), we then find the following maps of fields. First of all, the background charge of the field $\phi$ is shifted from $Q_{\phi}=b$ to $Q_{\phi}=b+1 / b$. After the twist, the field $\phi$ corresponds to the Liouville field. Recall that the central charge is written as $c=1+3 Q^{2}$ in terms of background charge $Q$. Next, the field $X$ would have background charge $Q_{X}=i(1 / b-b)$ after the twist, and this field becomes the bosonic part of the $\hat{c} \leq 1$ matter. The conformal weights of fermions $(\theta, p)$ are shifted from $(0,1)$ to $(1 / 2,1 / 2)$ and they become superpartners of the above bosonic fields. The other fields $\psi^{ \pm}$and $\varphi^{ \pm}$are mapped to the superghosts $(b, c)$ and $\left(\beta^{\prime}, \gamma^{\prime}\right)$ of type 0 superstring theory 6 In table 1 the changes of conformal weights are summarized. In the end we expect that $(\beta, \gamma)$ would be canceled out with $(B, C)$ as in the bosonic string case [14]. In this way we obtain the same field contents as the world-sheet theory of the type $0 \hat{c} \leq 1$ string including ghosts. More detailed explanation of the type 0 string will be given in subsection 4.1 .

\footnotetext{
${ }^{5}$ This choice means that we gauge the vector $U(1)$ current instead of the axial $U(1)$ current. The former may produce a trumpet like geometry and the latter a black hole like geometry [32. We choose the vector gauge just for the simplicity of expression.

${ }^{6}$ Here we use the notation $\left(\beta^{\prime}, \gamma^{\prime}\right)$ to represent superghosts of superstring in order to distinguish them from the ones in (2.3).
} 


\begin{tabular}{|c|c|c|c|c|}
\hline \multirow{2}{*}{} & \multicolumn{2}{|c|}{ Before twisting } & \multicolumn{2}{c|}{ After twisting } \\
\cline { 2 - 5 } & Central charge & Conformal weights & Central charge & Conformal weights \\
\hline$(\theta, p)$ & -2 & $(0,1)$ & 1 & $(1 / 2,1 / 2)$ \\
\hline$\left(\psi^{+}, \psi^{-}\right)$ & 1 & $(1 / 2,1 / 2)$ & -26 & $(2,-1)$ \\
\hline$\left(\varphi^{+}, \varphi^{-}\right)$ & -1 & $(1 / 2,1 / 2)$ & 11 & $(3 / 2,-1 / 2)$ \\
\hline$(\beta, \gamma)$ & 2 & $(1,0)$ & 2 & $(0,1)$ \\
\hline$(B, C)$ & -2 & $(1,0)$ & -2 & $(1,0)$ \\
\hline
\end{tabular}

Table 1: Changes of central charges and conformal weights after topological twisting.

\subsection{Chiral Primaries}

In the previous subsection we have shown that free fields in the $\mathcal{N}=2$ coset are mapped to the matter contents of the $\hat{c} \leq 1$ superstring theory after the topological twist. In fact we can identify physical operators of the topological model with those of the $\hat{c} \leq 1$ superstring, which is the subject of this subsection. In order to define the coset model we have introduced two spin $1 / 2$ fermions $\psi^{ \pm}$and two spin $1 / 2$ bosons $\varphi^{ \pm}$. With the bosonization formula, they can be written as

$$
\psi^{+}=e^{i H}, \quad \psi^{-}=e^{-i H}, \quad \varphi^{+}=e^{-\chi} \partial \xi, \quad \varphi^{-}=e^{\chi} \eta,
$$

where $H, \chi$ are free bosons without background charges and free fermions $\xi, \eta$ are with $\Delta_{\xi}=0, \Delta_{\eta}=1$. The non-trivial OPEs are given as

$$
H(z) H(0) \sim-\ln z, \quad \chi(z) \chi(0) \sim-\ln z, \quad \eta(z) \xi(0) \sim \frac{1}{z} .
$$

These bosonized expressions of fermions are useful to define vertex operators. Since the operators of the coset model must be invariant under the BRST charge (2.18), they should take the form

$$
e^{i r H+u \chi} \Phi_{j, m}^{s} e^{2 i b\left(m+r-\frac{u}{2}\right) X},
$$

whose conformal weight is

$$
\Delta=-2 b^{2} j\left(j+\frac{1}{2}\right)+\frac{s(s-1)}{2}+\frac{r^{2}}{2}-\frac{u^{2}}{2}+2 b^{2}\left(m+r-\frac{u}{2}\right)^{2} .
$$

Here we have used $\Phi_{j, m}^{s}$ as the vertex operator of OSP(1|2) WZNW model as defined in (2.8).

Physical operators of the topological model can be constructed from chiral primaries of the $\mathcal{N}=2$ coset model. Here we review how to perform the topological twist to the chiral primaries by following [14, 7]. First we find chiral primary states of the coset in 
NS-sector 7 which satisfy

$$
G_{r-1 / 2}^{+}|\mathrm{NS}\rangle=G_{r+1 / 2}^{-}|\mathrm{NS}\rangle=0
$$

for $r=0,1, \cdots$. Among the vertex operators of the form (2.23), there are chiral primary operators $\mathcal{O}_{j}^{N S, s}$ corresponding to the above chiral primary states. These chiral primaries can be mapped to R-ground states by spectral flow operation. Redefining the $\mathrm{U}(1)_{R}$ current (2.16) as

$$
J_{R}=-2 b^{2} \hat{J}^{3}-\psi^{+} \psi^{-}-\varphi^{+} \varphi^{-}=-i b \partial X_{R}
$$

with $X_{R}(z) X_{R}(0) \sim \ln z$, the R-ground states are obtained by $\mathcal{O}_{j}^{R, s}=e^{\frac{i}{2} b X_{R}} \mathcal{O}_{j}^{N S, s}$. Finally, the elements of cohomology for the topological theory are obtained by the topological twist as $\mathcal{O}_{j}^{s=1}=e^{-\frac{i}{2} \sqrt{k} b X_{R}^{\prime}} \mathcal{O}_{j}^{R, s}$. Here we define $X_{R}^{\prime}$ as

$$
J_{R}^{\prime}=-2 J^{3}-3 \psi^{+} \psi^{-}-2 \varphi^{+} \varphi^{-}+2 i b(k-2) \partial X=:-i \sqrt{k} b \partial X_{R}^{\prime}
$$

from the expression of R-current (2.20).

Among the chiral primaries of the $\mathcal{N}=2$ coset model, we focus on the following two types of operators in NS-sector;

$$
\mathcal{O}_{j}^{N S, \frac{1}{2}}=e^{-\frac{1}{2} \chi} \Phi_{j, j-\frac{1}{4}}^{\frac{1}{2}} e^{2 i b j X}, \quad \mathcal{O}_{j}^{N S, 1}=\Phi_{j, j}^{1} e^{2 i b j X},
$$

which satisfy $\Delta=q_{R} / 2=-j b^{2}-1 / 4$ and $\Delta=q_{R} / 2=-j b^{2}$, respectively. The R-sector ground states are constructed as

$$
\mathcal{O}_{j}^{R, \frac{1}{2}}=e^{\frac{i}{2} H} \Phi_{j, j-\frac{1}{4}}^{\frac{1}{2}} e^{2 i b\left(j+\frac{1}{4}\right) X}, \quad \mathcal{O}_{j}^{R, 1}=e^{\frac{i}{2} H+\frac{1}{2} \chi} \Phi_{j, j}^{1} e^{2 i b\left(j+\frac{1}{4}\right) X} .
$$

After the topological twist we finally obtain

$$
\mathcal{O}_{j}^{\frac{1}{2}}=e^{-i H-\chi} \Phi_{j, j-\frac{1}{4}}^{\frac{1}{2}, w=1} e^{2 i b\left(j+\frac{1}{4 b^{2}}\right) X}, \quad \mathcal{O}_{j}^{1}=e^{-i H-\frac{1}{2} \chi} \Phi_{j, j}^{1, w=1} e^{2 i b\left(j+\frac{1}{4 b^{2}}\right) X} .
$$

Notice that vertex operators are spectrally flowed in the sense of OSP(1|2) WZNW model as in (2.12) during the procedure of topological twist. Under the spectral flow action we may identify $\Phi_{j, j+\frac{s-1}{2}}^{s, w=1}=\Phi_{-j-\frac{k}{2}+\frac{1}{4}, j+\frac{s-1}{2}+\frac{k}{2}}^{s-\frac{1}{2}}$. Combining with the free field representation of vertex operators (2.8), we find

$$
\mathcal{O}_{j}^{\frac{1}{2}} \sim c e^{-\chi} e^{2 i b\left(j+\frac{1}{4 b^{2}}\right) X+2 b\left(j+\frac{k}{2}-\frac{1}{4}\right) \phi}, \quad \mathcal{O}_{j}^{1}=c e^{-\frac{1}{2} \chi} e^{\frac{i}{2} Y+2 i b\left(j+\frac{1}{4 b^{2}}\right) X+2 b\left(j+\frac{k}{2}-\frac{1}{4}\right) \phi} .
$$

\footnotetext{
${ }^{7}$ Notice that there are three different spin structures that appear in this paper. One is for the $\operatorname{OSP}(1 \mid 2)$ current algebra, which is defined such that an an integer $s$ in (2.8) means the NS-sector, while a half integer $s$ implies R-sector. The second spin structure is the ordinary one for the $N=2$ superconformal field theory. The third one is for the $\hat{c} \leq 1$ superstring. In this section the notion of NS,R is with respect to the second spin structure.
} 
In the above, we renamed $c=\exp (-i H)$ as suggested by the previous discussion. Moreover the $\beta$-ghost in the superstring should be written as $\beta^{\prime}=\partial \xi \exp (-\chi)$. Therefore, we can say that these operators have one $c$-ghost and picture -1.8

Notice that the above two operators (2.31) indeed coincide with the tachyon and $\mathrm{RR}$ field vertex operators in the two dimensional type 0 superstring, respectively (see subsection 4.1). Actually they complete the list of physical operators since there are no massive stringy modes in two dimensional superstring. This fact may be seen by taking the light-cone gauge. In this way, we have learned that the physical states (chiral primary states) in the topological string on $\operatorname{OSP}(1 \mid 2) / \mathrm{U}(1)$ are mapped into the physical states in the two dimensional type 0 string. We will study the relation between these two theories in more detail below.

\section{$3 \operatorname{OSP}(1 \mid 2) / \mathrm{U}(1)$ Coset from $\mathcal{N}=1$ Super Liouville}

In references [22, 23] it was shown that arbitrary correlation functions of primary fields in SL(2) WZNW model can be written in terms of correlation functions of Liouville field theory. This property may be useful to show the equivalence between the scattering amplitudes in $c \leq 1$ bosonic string and the topological string on $S L(2) / U(1)$. The agreement for three-point functions between them has been shown in [7], and this is generalized by [36] to arbitrary tree level amplitudes by utilizing the generalized relation of [37]. Recently it was shown in [21] that correlation functions of OSP(1|2) WZNW model can be written in terms of those of $\mathcal{N}=1$ super Liouville field theory. Later we would like to show the equivalence between $\mathcal{N}=2$ coset model of $\operatorname{OSP}(1 \mid 2) / \mathrm{U}(1)$ and the $\hat{c} \leq 1$ superstring in the level of amplitudes. For the purpose we generalize the relation such as to include RR-sectors of fermions and spectrally flowed sectors of $\operatorname{OSP}(1 \mid 2)$ model. In this section we derive the generalized relation in the path integral formulation following [23, 21].

\subsection{OSP(1|2) WZNW Model}

Let us start from the action of $\operatorname{OSP}(1 \mid 2)$ WZNW model. In terms of free fields the action may be written as 9

$$
\begin{aligned}
S^{\mathrm{WZNW}}(g)=\frac{1}{2 \pi} \int d^{2} z\left[\frac{1}{2} \partial \phi \bar{\partial} \phi\right. & +\frac{b}{8} \sqrt{g} \mathcal{R} \phi+\beta \bar{\partial} \gamma+\bar{\beta} \partial \bar{\gamma}+p \bar{\partial} \theta+\bar{p} \partial \bar{\theta} \\
& \left.-\frac{1}{k} \beta \bar{\beta} e^{2 b \phi}-\frac{1}{2 k}(p+\beta \theta)(\bar{p}+\bar{\beta} \bar{\theta}) e^{b \phi}\right],
\end{aligned}
$$

\footnotetext{
${ }^{8}$ The operators corresponding to those in the other picture may be obtained by the action of operator similar to the picture changing operator.

${ }^{9}$ A derivation of this action can be found in 21]. This action hare is a bit different from the one in [21], but it is easy to see the equivalence between the two expressions.
} 
where $\phi, \gamma, \bar{\gamma}, \theta, \bar{\theta}$ are related to the parameters of elements $g \in \operatorname{OSP}(1 \mid 2)$ and $\beta, \bar{\beta}, \theta, \bar{\theta}$ are conjugate variables. The generators of current algebra symmetry are written as in (2.3) in these variables. Here we use the form of vertex operator as

$$
V_{j}^{s, \bar{s}}(\mu \mid z)=\mu^{j+\frac{1}{2}+\frac{s}{2}} \bar{\mu}^{j+\frac{1}{2}+\frac{\bar{s}}{2}} e^{i s Y+i \bar{s} \bar{Y}} e^{\mu \gamma-\bar{\mu} \bar{\gamma}} e^{2 b\left(j+\frac{1}{2}\right) \phi} .
$$

For the NSNS-sector with $s, \bar{s}=0,1$ these vertex operators are the same as in [21]. The conformal weights are given as $\Delta=-2 b^{2} j(j+1 / 2)$. The vertex operators in the RR-sector are given by spin fields with $s=\bar{s}=1 / 2$, and the conformal weights are $\Delta=-2 b^{2} j(j+1 / 2)+1 / 8$. The above expression in so-called $\mu$-basis is useful for our purpose, and it can be mapped to the $m$-basis expression given in (2.8) by 10

$$
\Phi_{j, m, \bar{m}}^{s, \bar{s}}=\int \frac{d^{2} \mu}{|\mu|^{2}} \mu^{-m} \bar{\mu}^{-\bar{m}} V_{j}^{s, \bar{s}}(\mu \mid z) .
$$

In some sense, the $\mu$-basis expression can be thought of generating function of the $m$-basis expression.

Since the operators of topological model in (2.30) are written in terms of $\operatorname{OSP}(1 \mid 2)$ vertex operators with spectral flow index $w=1$, it is important to understand the symmetry under the spectral flow. The spectral flow action $\rho^{w}$ can be defined as

$$
\rho^{w}\left(J_{n}^{3}\right)=J_{n}^{3}-\frac{k}{2} w \delta_{n, 0}, \quad \rho^{w}\left(J_{n}^{ \pm}\right)=J_{n \pm w}^{ \pm}, \quad \rho^{w}\left(j_{r}^{ \pm}\right)=j_{r \pm \frac{w}{2}}^{ \pm},
$$

where the mode expansions are $J^{A}(z)=\sum_{n} J_{n}^{A} z^{-n-1}$ with $A= \pm, 3$ and $j^{ \pm}(z)=$ $\sum_{r} j_{r}^{ \pm} z^{-r-1}$. We can easily see that the new currents satisfy the same (anti-)commutation relations as before, which implies that the spectral flow is the symmetry of the current algebra $\operatorname{OSP}(1 \mid 2)$. The vacuum state is defined such as

$$
\rho^{w}\left(J_{n}^{A}\right)|w\rangle=0, \quad \rho^{w}\left(j_{r}^{ \pm}\right)|w\rangle=0
$$

for $n, r \geq 0$. In terms of free fields, the vacuum state $|w\rangle=|w\rangle_{(\beta, \gamma)} \otimes|w\rangle_{\phi} \otimes|w\rangle_{Y}$ is characterized as

$$
\beta_{n-w}|w\rangle_{(\beta, \gamma)}=0, \quad \gamma_{n+w}|w\rangle_{(\beta, \gamma)}=0
$$

for $n \geq 0$, and moreover

$$
|w\rangle_{\phi}=e^{\frac{w}{2 b} \phi}|0\rangle_{\phi}, \quad|w\rangle_{Y}=e^{-\frac{i w}{2} Y}|0\rangle_{Y}
$$

In the following we assume $w \geq 0$ and denote $v^{w}(0)$ as the operator corresponding to the state $|w\rangle$.

\footnotetext{
${ }^{10}$ In order to compare with the previous notation, we may need to perform a flip $j \rightarrow-j-1 / 2$. Moreover, it might be natural to multiply the factor $N_{j, m, \bar{m}}^{s . \bar{s}}=\frac{\Gamma(-j+1 / 2-s / 2+m)}{\Gamma(j+1 / 2+\bar{s} / 2-\bar{m})}$ as, e.g., in [22]; see also [38. Here we remove it since it may diverge in our case.
} 
As discussed in [37, 39], generic $N$-point functions of operators with spectral flow can be reduced to $N$-point functions of $(3.2)$ with the inversion of $v^{w}(\xi)$. We choose the position of insertion $v^{w}(\xi)$ as $\xi=0$ since it does not affect the following discussion. In the path integral formulation they are given as

$$
\begin{aligned}
\left\langle\prod_{\nu=1}^{N} V_{j_{\nu}}^{s_{\nu} \bar{s}_{\nu}}\left(\mu_{\nu} \mid z_{\nu}\right) v^{w}(0)\right\rangle= & \int_{(w)} \mathcal{D} \phi \mathcal{D}^{2} \beta \mathcal{D}^{2} \gamma \mathcal{D}^{2} \theta \mathcal{D}^{2} p e^{-S^{\mathrm{WZNW}}(g)} \times \\
& \times \prod_{\nu=1}^{N} V^{s_{\nu}, \bar{s}_{\nu}}\left(\mu_{\nu} \mid z_{\nu}\right) e^{w(\phi(0) / 2 b-i Y(0) / 2)} .
\end{aligned}
$$

The effects of insertion $v^{w}(0)$ appears in the right hand side in two ways. One is the extra insertion of vertex operator $e^{w(\phi(0) / 2 b-i Y(0) / 2)}$, and the other is the restriction to the integration domain of $\beta, \bar{\beta}$ such that $\beta, \bar{\beta}$ have a zero of order $w$ at $\xi=0$. For more detail see 39 .

\subsection{OSP (1|2)-Super Liouville Correspondence}

Now that we have the $\operatorname{OSP}(1 \mid 2)$ WZNW model, we can derive the relation between the correlation functions of $\operatorname{OSP}(1 \mid 2)$ WZNW model and those of $\mathcal{N}=1$ super Liouville field theory by following the analysis of [21]. For this purpose we first integrate $\beta, \gamma$ as in [21]. Integrations over $\gamma$ and $\bar{\gamma}$ lead to delta functionals for $\beta$ and $\bar{\beta}$, which replace the fields $\beta, \bar{\beta}$ by

$$
\beta(x)=\sum_{\nu=1}^{N} \frac{\mu_{\nu}}{x-z_{\nu}}=u \frac{x^{w} \prod_{i=1}^{N-2-w}\left(x-y_{i}\right)}{\prod_{\nu=1}^{N}\left(x-z_{\nu}\right)}=: u \mathcal{B}(x) .
$$

The insertion of $v^{w}(0)$ forces $\beta(x)$ to have a zero of order $w$ at $x=0$ and this requirement gives constraints

$$
\sum_{\nu=1}^{N} \mu_{\nu} z_{\nu}^{-n}=0
$$

for $n=0,1, \cdots, w$. Since a 1 -form with $N$ poles on a sphere has $N-2$ zero's, $\beta$ can be represented as in the right hand side by the positions of $N-2-w$ more zero's $y_{i}$. In other words, the parameters $y_{i}$ are defined by the equation (3.9), and the new parameters are essential to relate the model to super Liouville theory as seen below. Moreover, we can see that the number of spectral flow $w$ is restricted as $w \leq N-2$.

After the integration over $\beta, \gamma$, the action becomes something similar to super Liouville theory, but the coefficients include functions $\mathcal{B}(z), \overline{\mathcal{B}}(\bar{z})$. Following [21, 39] these can be removed by the redefinition of fields as

$$
\phi^{\prime}:=\phi+\frac{1}{2 b} \ln |u \mathcal{B}|^{2}, \quad Y^{\prime}:=Y-\frac{i}{2} \ln |u \mathcal{B}|^{2} .
$$


Moreover, after some manipulations we find the relation

$$
\begin{aligned}
& \left\langle\prod_{\nu=1}^{N} V_{j_{\nu}}^{s_{\nu}, \bar{s}_{\nu}}\left(\mu_{\nu} \mid z_{\nu}\right) v^{w}(0)\right\rangle \\
& \quad=\prod_{n=0}^{w} \delta^{2}\left(\sum_{\nu} \mu_{\nu} z^{-n}\right)|u|^{2-\frac{w}{2 b^{2}}+\frac{w}{2}}\left|\Theta_{N}^{w}\right|^{2}\left\langle\prod_{\nu=1}^{N} V_{\alpha_{\nu}}^{s_{\nu}-\frac{1}{2}, \bar{s}_{\nu}-\frac{1}{2}}\left(z_{\nu}\right) \prod_{j=1}^{N-2-w} V_{-\frac{1}{2 b}}^{\frac{1}{2}, \frac{1}{2}}\left(y_{j}\right)\right\rangle
\end{aligned}
$$

with $\alpha_{\nu}=2 b\left(j_{\nu}+1 / 2\right)+1 / 2 b$. The right hand side is computed by the sum of $\mathcal{N}=1$ super Liouville theory $\left(\phi^{\prime}, \psi, \bar{\psi}\right)$ and massless fermions $\left(\psi_{X}, \bar{\psi}_{X}\right)$

$$
\begin{aligned}
S\left[\phi^{\prime}, \psi, \psi_{X}\right]=\frac{1}{4 \pi} \int d^{2} z & {\left[\partial \phi^{\prime} \bar{\partial} \phi^{\prime}+\frac{Q_{\phi^{\prime}}}{4} \sqrt{g} R \phi^{\prime}+\frac{2}{k} e^{2 b \phi^{\prime}}+\right.} \\
& \left.+\psi \bar{\partial} \psi+\bar{\psi} \partial \bar{\psi}+\psi_{X} \bar{\partial} \psi_{X}+\bar{\psi}_{X} \partial \bar{\psi}_{X}-\frac{2}{k} \psi \bar{\psi} e^{b \phi^{\prime}}\right]
\end{aligned}
$$

with $Q_{\phi^{\prime}}=b+1 / b$. The fermions are defined by

$$
\psi \pm i \psi_{X}=\sqrt{2} e^{ \pm i Y^{\prime}}, \quad \bar{\psi} \pm i \bar{\psi}_{X}=\sqrt{2} e^{ \pm i \bar{Y}^{\prime}}
$$

and the vertex operators are

$$
V_{\alpha}^{s, \bar{s}}(z)=e^{i s Y+i s \bar{Y}} e^{\alpha \phi^{\prime}}
$$

with conformal weights $\Delta=\alpha\left(Q_{\phi^{\prime}}-\alpha\right) / 2+s^{2} / 2$. The twist factor is

$$
\Theta_{N}^{w}=\prod_{\mu<\nu}^{N}\left(z_{\mu}-z_{\nu}\right)^{\frac{1}{4 b^{2}}-\frac{1}{4}} \prod_{i<j}^{N-2-w}\left(y_{i}-y_{j}\right)^{\frac{1}{4 b^{2}}-\frac{1}{4}} \prod_{\nu=1}^{N} \prod_{i=1}^{N-2-w}\left(z_{\nu}-y_{j}\right)^{-\frac{1}{4 b^{2}}+\frac{1}{4}}
$$

Here we should notice that the operators in the NSNS(RR)-sector are mapped to those in the RR(NSNS)-sector. Moreover, if the winding number is violated maximally as $w=N-2$, then there is no extra insertion of operator at $z=y_{i}$.

\subsection{Amplitudes of OSP(1|2)/U(1) Coset Model}

Utilizing the fundamental relation (3.12), we can rewrite correlation functions of $\operatorname{OSP}(1 \mid 2) / \mathrm{U}(1)$ coset in terms of $\mathcal{N}-1$ super Liouville theory with a supersymmetric pair of free boson and fermion in a manner similar to the bosonic case [39]. Here the vertex operators of coset model are defined as in (2.11) 11

$$
\Psi_{j, m, \bar{m}}^{s, \bar{s}}(z, \bar{z})=V_{m, \bar{m}}^{X^{3}}(z, \bar{z}) \Phi_{j, m, \bar{m}}^{s, \bar{s}}(z, \bar{z})
$$

\footnotetext{
${ }^{11}$ Only in this subsection we construct the coset model by gauging the axial U(1) symmetry in order to use the trick of [39].
} 
with

$$
V_{m, \bar{m}}^{X^{3}}(z, \bar{z})=e^{i \sqrt{\frac{2}{k}}\left(-m X^{3}+\bar{m} \bar{X}^{3}\right)}
$$

Moreover, the vertex operators of $\operatorname{OSP}(1 \mid 2)$ model with spectral flow index $w$ are related to vertex operators of $\operatorname{OSP}(1 \mid 2) / \mathrm{U}(1)$ model as (see (2.12))

$$
\Psi_{j, m, \bar{m}}^{s, \bar{s}}(z, \bar{z})=V_{m+\frac{k w}{2}, \bar{m}+\frac{k w}{2}}^{X^{3}}(z, \bar{z}) \Phi_{j, m, \bar{m}}^{s, \bar{s}, w}(z, \bar{z}) .
$$

Therefore we can also obtain a formula for correlation functions of OSP(1|2) model with non-trivial spectral flow actions. In particular, we will be interested in a specific $N$-point function in $\operatorname{OSP}(1 \mid 2)$ WZNW model as

$$
\mathcal{M}=\left\langle\Phi_{j_{1}, m_{1}, \bar{m}_{1}}^{s_{1}, \bar{s}_{1}}\left(z_{1}\right) \Phi_{j_{2}, m_{2}, \bar{m}_{2}}^{s_{2}, \bar{s}_{2}}\left(z_{2}\right) \prod_{\nu=3}^{N} \Phi_{j_{\nu}, m_{\nu}, \bar{m}_{\nu}}^{s_{\nu}, \bar{s}_{\nu}, w_{\nu}=1}\left(z_{\nu}\right)\right\rangle .
$$

Since the amplitude has $N-2$ number of winding violation, it should be written in terms of $N$-point function of $\mathcal{N}=1$ super Liouville theory.

Let us first study $N$-point function of $\operatorname{OSP}(1 \mid 2) / \mathrm{U}(1)$ coset model. As before we introduce a new field $\hat{X}^{3}$ by

$$
\hat{X}_{L}^{3}:=X_{L}^{3}-i \sqrt{\frac{k}{2}} \ln (u \mathcal{B})
$$

and the right mover defined by its complex conjugate. By closely following [23] and utilizing the formula (3.12), we finally obtain

$$
\begin{aligned}
& \left\langle\prod_{\nu=1}^{N} \Psi_{j_{\nu}, m_{\nu}, \bar{m}_{\nu}}^{s_{\nu}, \bar{s}_{\nu}}\right\rangle=\int \frac{\prod_{i=1}^{N-2-w} d^{2} y_{i}}{(N-2-w) !} \times \\
& \quad \times\left\langle\prod_{\nu=1}^{N} V_{m_{\nu}+\frac{k}{2}, \bar{m}_{\nu}+\frac{k}{2}}^{\hat{X}^{3}}\left(z_{\nu}\right) V_{\alpha_{\nu}}^{s_{\nu}-\frac{1}{2}, \bar{s}_{\nu}-\frac{1}{2}}\left(z_{\nu}\right) \prod_{i=1}^{N-2-w} V_{-\frac{k}{2},-\frac{k}{2}}^{\hat{X}^{3}}\left(y_{i}\right) V_{-\frac{1}{2 b}}^{\frac{1}{2}, \frac{1}{2}}\left(y_{i}\right)\right\rangle .
\end{aligned}
$$

The label $w$ is related to the winding number violation as $\sum_{\nu} m_{\nu}=\sum_{\nu} \bar{m}_{\nu}=-\frac{k w}{2}$. The right hand side should be computed by the theory with the action $S\left[\phi^{\prime}, \psi, \psi_{X}\right]$ for $\mathcal{N}=1$ super Liouville theory and a free fermion $\left(\psi_{X}, \bar{\psi}_{X}\right)$ and a free boson $\hat{X}^{3}$ with background charge $Q=-i \sqrt{k}$ for its dual field.

With the formula (3.22) and (3.19) we can write down generic correlation functions in the OSP $(1 \mid 2)$ model with spectral flow action considered in terms of super Liouville theory. Here we only compute the amplitude (3.20) since it is the case used in the later analysis. With the formula (3.19) we can relate the amplitude (3.20) to a $N$-point function of the coset as

$$
\left\langle\prod_{\nu=1}^{N} \Psi_{j_{\nu}, m_{\nu}, \bar{m}_{\nu}}^{s_{\nu}, \bar{s}_{\nu}}\right\rangle=\mathcal{M} \times\left\langle V_{m_{1}, \bar{m}_{1}}^{X^{3}}\left(z_{1}\right) V_{m_{2}, \bar{m}_{2}}^{X^{3}}\left(z_{2}\right) \prod_{\nu=3}^{N} V_{m_{\nu}+\frac{k}{2}, \bar{m}_{\nu}+\frac{k}{2}}^{X^{3}}\left(z_{\nu}\right)\right\rangle .
$$


Then, by combining with the formula (3.22), we find

$$
\mathcal{M}=\left|\Theta_{s}\left(z_{\nu}\right)\right|^{2}\left\langle\prod_{\nu=1}^{N} V_{\alpha_{\nu}}^{s_{\nu}-\frac{1}{2}, \bar{s}_{\nu}-\frac{1}{2}}\left(z_{\nu}\right)\right\rangle,
$$

where the right hand side is computed by the $\mathcal{N}=1$ super Liouville theory and a free fermion with the action (3.13). The coefficient is given by

$$
\Theta_{s}\left(z_{\nu}\right)=\left(z_{1}-z_{2}\right)^{\frac{k}{2}+m_{1}+m_{2}} \prod_{\nu=3}^{N}\left[\left(z_{1}-z_{\nu}\right)\left(z_{2}-z_{\nu}\right)\right]^{\frac{k}{2}+m_{\nu}},
$$

and bared expression for $\bar{\Theta}_{s}\left(\bar{z}_{\nu}\right)$. This formula will be important to relate amplitudes of the topological model and the $\hat{c} \leq 1$ superstring theory.

\section{Correspondence to $\hat{c} \leq 1$ Superstring Theory}

In section 2, we have studied the topological model based on the $\mathcal{N}=2$ supersymmetric coset $\operatorname{OSP}(1 \mid 2) / \mathrm{U}(1)$. In particular, we have observed that free fields and chiral primaries of the coset model are mapped to matter contents and physical operators in the $\hat{c} \leq 1$ superstring theory. In this section, we establish the relation in more detail. After briefly reviewing the $\hat{c} \leq 1$ superstring theory and the method to compute amplitudes in topological models, we compare the amplitudes of both theories.

\section{$4.1 \hat{c} \leq 1$ Superstring Theory}

In section 2 we have already encountered the $\hat{c} \leq 1$ superstring theory during constructing the topological model of the coset $\operatorname{OSP}(1 / 2) / \mathrm{U}(1)$. In this subsection we define the $\hat{c} \leq 1$ superstring theory in a more precise way 12 The matter part consists of a linear dilaton $X$ with background charge $Q_{X}=i(1 / b-b)$ and a free fermion $\psi_{X}$. The action of these fields is given by

$$
S_{X}=\frac{1}{4 \pi} \int d^{2} z\left[\partial X \bar{\partial} X+\frac{Q_{X}}{4} \sqrt{g} \mathcal{R} X+\psi_{X} \bar{\partial} \psi_{X}+\bar{\psi}_{X} \partial \bar{\psi}_{X}\right] .
$$

The theory also includes the $\mathcal{N}=1$ super Liouville theory, whose action is

$$
S=\frac{1}{4 \pi} \int d^{2} z\left[\partial \phi \bar{\partial} \phi+\frac{Q_{\phi}}{4} \sqrt{g} \mathcal{R} \phi+\psi \bar{\partial} \psi+\bar{\psi} \partial \bar{\psi}+\mu_{L} \psi \bar{\psi} e^{b \phi}\right]
$$

with $Q_{\phi}=b+1 / b$. The total central charge is now $c=3 / 2+3 Q_{X}^{2}+3 / 2+3 Q_{\phi}^{2}=15$, and hence we can construct a critical superstring theory by coupling the world-sheet superghosts $(b, c)$ and $\left(\beta^{\prime}, \gamma^{\prime}\right)$.

Primary operators of this theory may take the form $\exp (\alpha X+\beta \phi)$, which has the conformal weight $\Delta=\alpha\left(Q_{X}-\alpha\right) / 2+\beta\left(Q_{\phi}-\beta\right) / 2$. Following the standard method

\footnotetext{
${ }^{12}$ Notice that we are setting $\alpha^{\prime}=2$ in this paper.
} 
to construct BRST invariant operators, we can find out physical operators in the $\hat{c} \leq 1$ superstring theory. The tachyon vertex operator is given as

$$
c \bar{c} \mathcal{T}_{p}^{(-1)}=c \bar{c} e^{-(\chi+\bar{\chi})} e^{i k_{X}(X+\bar{X})+k_{\phi}^{ \pm} \phi}
$$

where the momenta run over $i k_{X}=Q_{X} / 2+i p$ and $k_{\phi}^{ \pm}=Q_{\phi} / 2 \pm p$ with $p \in \mathbb{R}$. We bosonize the superghost $\beta^{\prime}, \gamma^{\prime}$ like in (2.21), which yields the new fields $\chi$. In other words, the above expression is in $(-1,-1)$ picture; and in $(0,0)$ picture it is written as

$$
c \bar{c} \mathcal{T}_{p}^{(0)}=c \bar{c}\left(i k_{X} \psi_{X}+k_{\phi}^{ \pm} \psi\right)\left(i k_{X} \bar{\psi}_{X}+k_{\phi}^{ \pm} \bar{\psi}\right) e^{i k_{X}(X+\bar{X})+k_{\phi}^{ \pm} \phi} .
$$

There are other physical operators in the RR-sector. The Ramond vertex operator in $(-1 / 2,-1 / 2)$ picture is written as

$$
c \bar{c} \mathcal{R}_{p}^{(-1 / 2)}=c \bar{c} e^{-\frac{1}{2}(\chi+\bar{\chi})} e^{ \pm \frac{i}{2}(Y+\bar{Y})+i k_{X}\left(X_{L}+X_{R}\right)+k_{\phi}^{ \pm} \phi} .
$$

Indeed, these vertex operators (4.3) and (4.5) are the same as those obtained from the topological string on $\operatorname{OSP}(1 \mid 2) / \mathrm{U}(1)$ as observed in (2.31).

If $X$ direction is compactified with radius $R$, then the momentum takes discrete values $p=n / R$ with $n \in \mathbb{Z}$. For winding modes we should replace $X_{R} \rightarrow-X_{R}, \bar{H} \rightarrow-\bar{H}$ and $p=w R / 2$ with $w \in \mathbb{Z}$. After the proper GSO projection, type 0B theory has the tachyon modes and the momentum modes in the RR-sector, On the other hand, type 0A theory has the tachyon modes and the winding modes in the RR-sector. See, e.g. [4, 5] for more detail.

\subsection{Amplitudes of Topological Model}

Before dealing with the specific case of $\operatorname{OSP}(1 \mid 2) / \mathrm{U}(1)$, we give generic arguments on amplitudes in topological models. Let us consider a topological field theory obtained by the topological twist $T^{t o p}(z)=T(z)+1 / 2 \partial J_{R}(z)$ of a $\mathcal{N}=2$ super conformal field theory [34, 35]. We consider the B model, namely twist the same way for the anti-holomorphic part as $\bar{T}^{t o p}(\bar{z})=\bar{T}(\bar{z})+1 / 2 \bar{\partial} \bar{J}_{R}(\bar{z})$. Then the physical spectrum can be computed from the cohomology of BRST operator $Q=\oint G^{+}(z) d z$. Let us write a basis of physical operators (in NS sector) as $\phi_{i}$, then we can obtain other types of physical operators as

$$
\oint d z G_{-\frac{1}{2}}^{-} \cdot \phi_{i}, \quad \oint d \bar{z} \bar{G}_{-\frac{1}{2}}^{-} \cdot \phi_{i}, \quad \int d^{2} z G_{-\frac{1}{2}}^{-} \bar{G}_{-\frac{1}{2}}^{-} \cdot \phi_{i} .
$$

Following the arguments on [40, 35, 41], we compute amplitudes of the form

$$
\mathcal{F}=\left\langle\phi_{i_{1}}\left(z_{1}\right) \phi_{i_{2}}\left(z_{2}\right) \phi_{i_{3}}\left(z_{3}\right) \prod_{\nu=4}^{N}\left[\int d^{2} z_{\nu} \tilde{\phi}_{i_{\nu}}\left(z_{\nu}\right)\right]\right\rangle
$$

which would give us non-trivial information of the topological model. Here we have defined $\tilde{\phi}_{i}=G_{-\frac{1}{2}}^{-} \bar{G}_{-\frac{1}{2}}^{-} \phi_{i}$. 
An important fact is that the above amplitudes of topological model can be computed in the original untwisted model [35]. After the topological twist the $U(1)_{R}$ current becomes anomalous, and hence we should insert $U(1)_{R}$ fields into correlators of original model to reproduce the topological amplitudes. Here we insert the operator $\mu(z, \bar{z})=e^{\left.\frac{i}{2} \sqrt{\frac{c}{3}}\left(X_{R}(z)\right)+\bar{X}_{R}(\bar{z})\right)}$ at two points $z=z_{1}, z_{2}$, where the free boson $X_{R}$ is related to the R-current as $J_{R}(z)=-i \sqrt{\frac{c}{3}} \partial X_{R}(z)$. This operator maps the physical operators $\phi_{i}$ of the topological model to operators $\phi_{i}^{R}$ in the R-ground states of the original model. In this way, we can write the topological amplitude (4.7) as

$$
\begin{aligned}
\mathcal{F} & =\left|z_{1}-z_{2}\right|^{q_{1}+q_{2}}\left(\left|z_{1}-z_{3}\right|\left|z_{2}-z_{3}\right|\right)^{q_{3}} \times \\
& \times\left\langle\phi_{i_{1}}^{R}\left(z_{1}\right) \phi_{i_{2}}^{R}\left(z_{2}\right) \phi_{i_{3}}\left(z_{3}\right) \prod_{\nu=4}^{N}\left[\int d^{2} z_{\nu}\left(\left|z_{1}-z_{\nu}\right|\left|z_{2}-z_{\nu}\right|\right)^{q_{\nu}-1} \tilde{\phi}_{i_{\nu}}\left(z_{\nu}\right)\right]\right\rangle,
\end{aligned}
$$

where the right hand side is computed in the original model before the topological twisting. Here we have denoted $q_{\nu}$ as the $U(1)_{R}$ charge of $\phi_{i_{\nu}}$.

\subsection{Comparison of Correlation Functions}

After the preparation we can now compare correlation functions of topological model on $\operatorname{OSP}(1 \mid 2) / \mathrm{U}(1)$ and of the $\hat{c} \leq 1$ superstring. We start from the amplitude of the topological model, and then show the equivalence by using the formula (3.24) obtained above. Here we only consider the amplitudes of operator of the first type in (2.30), which corresponds to the tachyon operator in the $\hat{c} \leq 1$ superstring. The case with the second type in (2.30) can be analyzed in a similar way.

Since the conservation of $\mathrm{U}(1)$ current $J=\partial \chi$ is violated by the amount of -2 , non vanishing amplitudes may be given as

$$
\mathcal{F}=\left\langle\mathcal{O}_{j_{1}}^{\frac{1}{2}}\left(z_{1}\right) \mathcal{O}_{j_{2}}^{\frac{1}{2}}\left(z_{2}\right) \mathcal{O}_{j_{3}}^{-\frac{1}{2}}\left(z_{3}\right) \prod_{\nu=4}^{N}\left[\int d^{2} z_{\nu} \tilde{\mathcal{O}}_{j_{\nu}}^{-\frac{1}{2}}\left(z_{\nu}\right)\right]\right\rangle
$$

This violation corresponds to the fact that the sum of picture must be -2 in superstring theory. The operator $\mathcal{O}_{j}^{\frac{1}{2}}$ is defined in (2.30) as

$$
\mathcal{O}_{j}^{\frac{1}{2}}=e^{-i(H+\bar{H})-(\chi+\bar{\chi})} \Phi_{j, j-\frac{1}{4}, j-\frac{1}{4}}^{\frac{1}{2}, \frac{1}{2}, w=1} e^{2 i b\left(j+\frac{1}{4 b^{2}}\right)(X+\bar{X})} .
$$

Following the argument in the previous subsection, this operator would be mapped to the R-ground state operator of the original model

$$
\mathcal{O}_{j}^{R, \frac{1}{2}}=e^{\frac{i}{2}(H+\bar{H})} \Phi_{j, j-\frac{1}{4}, j-\frac{1}{4}}^{\frac{1}{2}, \frac{1}{2}} e^{2 i b\left(j+\frac{1}{4}\right)(X+\bar{X})} .
$$

Another operator $\mathcal{O}_{j}^{-\frac{1}{2}}$ is given by a linear combination of

$$
e^{-i(H+\bar{H})} \Phi_{j, j+\frac{1}{4}, j+\frac{1}{4}}^{s, \bar{s}, w=1} e^{2 i b\left(j+\frac{1}{4 b^{2}}\right)(X+\bar{X})}
$$


with $s, \bar{s}=-1 / 2,3 / 2$. This operator should correspond to picture $(0,0)$ tachyon, and can be constructed by following the analysis in section 2, The other operator $\tilde{\mathcal{O}}_{j}^{-\frac{1}{2}}$ is then generated by the action of $G_{-1 / 2}^{-} \bar{G}_{-1 / 2}^{-}$as mentioned before and written as a linear combination of

$$
\Phi_{j, j-\frac{3}{4}, j-\frac{3}{4}}^{s, \bar{s}, w=1} e^{2 i b\left(j+\frac{1}{4 b^{2}}\right)(X+\bar{X})}
$$

with $s, \bar{s}=-1 / 2,3 / 2$.

As argued in the previous subsection, we first map the amplitude of topological model (4.9) to that of original model before twisting as in (4.8). Then we can use the formula (3.24) to relate it to the amplitude of $\mathcal{N}=1$ super Liouville theory. Following the analysis of [36, 7] we can then show that

$$
\mathcal{F}=\left\langle c \bar{c} \mathcal{T}_{j_{1}}^{(-1)}\left(z_{1}\right) c \bar{c} \mathcal{T}_{j_{2}}^{(-1)}\left(z_{2}\right) c \bar{c} \mathcal{T}_{j_{3}}^{(0)}\left(z_{3}\right) \prod_{\nu=4}^{N}\left[\int d^{2} z_{\nu} \mathcal{T}_{j_{\nu}}^{(0)}\left(z_{\nu}\right)\right]\right\rangle
$$

up to some coefficients. Here, the operators $\mathcal{T}^{(p)}$ are tachyon operators in the $p$-th picture and they are given by

$$
\begin{aligned}
& \mathcal{T}_{p}^{(-1)}=e^{-(\chi+\bar{\chi})} e^{i k_{X}(X+\bar{X})+k_{\phi}^{+} \phi} \\
& \mathcal{T}_{p}^{(0)}=\left(i k_{X} \psi_{X}+k_{\phi}^{+} \psi\right)\left(i k_{X} \bar{\psi}_{X}+k_{\phi}^{+} \bar{\psi}\right) e^{i k_{X}(X+\bar{X})+k_{\phi}^{+} \phi}
\end{aligned}
$$

with $k_{X}=(1 / b-b) / 2+2 b(j+1 / 4)$ and $k_{\phi}^{+}=(1 / b+b) / 2+2 b(j+1 / 4)$. In this way we have shown that the amplitude of topological string on $\operatorname{OSP}(1 \mid 2) / \mathrm{U}(1)$ can be identified with that of the $\hat{c} \leq 1$ superstring.

\section{Conclusion and Discussions}

In this paper we have proposed an equivalence between the topological string theory based on the coset $\operatorname{OSP}(1 \mid 2) / \mathrm{U}(1)$ and the $\widehat{c} \leq 1$ superstring theory. The latter is constructed by coupling a $\hat{c} \leq 1$ matter to the $\mathcal{N}=1$ super Liouville theory. This can be regarded as a supersymmetric version of the equivalence between the topological string on $S L(2) / U(1)$ and the $c \leq 1$ bosonic string, which was originally discovered by Mukhi and Vafa [14] for the case $c=1$ and was later generalized to the $c<1$ case in [7]. First we showed in the free field description that the field contents and the physical operators of the world-sheet theories of both string theories match. Moreover, we investigated the proposed equivalence at the level of scattering amplitudes by applying the map [21] between correlation functions in the $\operatorname{OSP}(1 \mid 2)$ WZNW model and in super Liouville field theory.

This map is a supersymmetric version of the one found by Ribault and Teschner to relate correlation functions in the $\mathrm{SL}(2)$ WZNW model and those in Liouville theory 
[22, 23]. In the last years, the result has been used with great success to investigate different dualities between non-rational conformal field theories. In particular, it has led to the proof of Fateev-Zamolodchikov-Zamolodchikov conjecture in [39]. The duality between different non-rational two-dimensional conformal field theories has a long story, and now a considerable list of examples is available: quantum Hamiltonian reduction [16], Mukhi-Vafa duality [14, 7], and Fateev-Zamolodchikov-Zamolodchikov duality [42] (and its supersymmetric version [43]) are probably the most renowned examples. These examples were, in fact, very useful to study string theory. For instance, it was the FateevZamolodchikov-Zamolodchikov duality what really permitted to construct a dual matrix model for strings in the the 2D black hole background 44. It is our hope that the new equivalence between conformal theories we studied in this paper will be relevant to understand new aspects of superstring dualities as well.

There are a number of issues which should be understood in the future. First we would like to understand better the spin structure and the picture changing operation of the topological string theory on the supercoset. It is also important to prove the complete equivalence of physical states between these two theories. An exhaustive analysis of the cohomology of the theory is needed to this end. Finally, it would be nice if we could understand a geometrical interpretation of the supercoset $\mathrm{OSP}(1 \mid 2) / \mathrm{U}(1)$ in terms of a certain (maybe super) Calabi-Yau manifold, as $\mathrm{SL}(2) / \mathrm{U}(1)$ coset model is related to the conifold.

\section{Acknowledgement}

We are very grateful to K. Hori for a useful discussion. GG thanks the members of the IPMU for their hospitality during his stay. YH would like to thank T. Creutzig, H. Irie and P. B. Rønne for useful discussions. The work of GG has been partially supported by ANPCyT grant PICT-2007-00849, by UBACyT grants X861 and X432, and by JSPSCONICET cooperation programme. The work of YH is supported by JSPS Research Fellowship. TT is supported by World Premier International Research Center Initiative (WPI Initiative), MEXT, Japan. The work of TT is supported in part by JSPS Grant-inAid for Scientific Research No.20740132 and by JSPS Grant-in-Aid for Creative Scientific Research No. 19GS0219.

\section{A Free Field Correlation Functions}

Although the computation of $N$-point functions in the OSP(1|2) WZNW model also involves fermion contributions and the insertion of picture changing operators, the building blocks to construct such observables are the correlation functions of vertices (2.8). Let us discuss these correlation functions in the free field representation proposed here. 
Consider the vertex operators

$$
\Phi_{j, m}^{s}(z)=N_{j, m}^{s} \gamma_{(z)}^{-j-1 / 2+m-s / 2} e^{2(j+1 / 2) b \phi(z)} e^{i s Y(z)} \times h . c .
$$

where h.c. stands for the anti-holomorphic portion of the operator 13 and $N_{j, m}^{s}$ refers to the normalization.

Correlation functions are defined as follows;

$$
\left\langle\prod_{\nu=1}^{N} \Phi_{j_{n}, m_{\nu}}^{s_{\nu}}\left(z_{\nu}\right)\right\rangle=\int \mathcal{D} \phi \mathcal{D}^{2} \beta \mathcal{D}^{2} \gamma \mathcal{D}^{2} \theta \mathcal{D}^{2} p e^{-S^{\mathrm{WZNW}}(g)} \prod_{\nu=1}^{N} \Phi_{j_{n}, m_{\nu}}^{s_{\nu}}\left(z_{\nu}\right)
$$

where $S^{\text {WZNW }}(g)$ refers to the action of the WZNW model (3.1). It is convenient to consider again the bosonization (2.5); that is, defining $\theta=e^{i Y}, p=e^{-i Y}$. The existence of non-trivial background charges associated to the fields $\phi$ and $Y$ requires special treatment of correlators. As usual in the Coulomb gas representation, this charge compensation is achieved by inserting additional operators that contribute to screen the charges at infinity. Screening operators are exact marginal deformations of the affine theory. In this theory four operators of this kind are available; namely

$$
\begin{array}{ll}
\mathcal{S}_{++}(z, \bar{z})=\frac{\lambda}{2 k} S_{+}(z) \bar{S}_{+}(\bar{z}), & \mathcal{S}_{+-}(z, \bar{z})=\frac{\lambda}{2 k} S_{+}(z) \bar{S}_{-}(\bar{z}), \\
\mathcal{S}_{-+}(z, \bar{z})=\frac{\lambda}{2 k} S_{-}(z) \bar{S}_{+}(\bar{z}), & \mathcal{S}_{--}(z, \bar{z})=\frac{\lambda}{2 k} S_{-}(z) \bar{S}_{-}(\bar{z}),
\end{array}
$$

with

$$
S_{+}(z)=e^{-i Y(z)} e^{b \phi(z)}, \quad S_{-}(z)=\beta_{(z)} e^{+i Y(z)} e^{b \phi(z)},
$$

and where $\lambda$ is a constant (see below).

The $N$-point correlation functions are thus defined by inserting different amount of screening operators (A.2)-(A.3) in the correlators, in addition to the $N$ vertex operators. Non-vanishing correlation functions are given by

$$
\begin{aligned}
& n_{+}-n_{-}=\sum_{\nu=1}^{N} s_{\nu}-1, \quad \bar{n}_{+}-\bar{n}_{-}=\sum_{\nu=1}^{N} \bar{s}_{\nu}-1 \\
& n_{+}+n_{-}=\bar{n}_{+}+\bar{n}_{-}=-2 \sum_{\nu=1}^{N} j_{\nu}+1-N
\end{aligned}
$$

together with

$$
\sum_{\nu=1}^{N} m_{\nu}=\sum_{\nu=1}^{N} \bar{m}_{\nu}=0
$$

where $n_{ \pm}\left(\right.$and $\left.\bar{n}_{ \pm}\right)$are the amount of operators of the type $S_{ \pm}(z)\left(\operatorname{resp} . \bar{S}_{ \pm}(\bar{z})\right)$ in the correlators. Equations (A.5)-(A.7) determine the amount of screening operators in terms of the quantum number of the vertices involved in the correlators.

\footnotetext{
${ }^{13}$ More precisely, the h.c. refers to the "bared contribution", and not necessarily to the anti-holomorphic part. Actually, labels $s, m$ and $\bar{s}, \bar{m}$ are not necessarily related by complex conjugation.
} 
To illustrate the Coulomb gas prescription, let us consider the sector $s_{\nu}=\bar{s}_{\nu}$, which yields $n_{ \pm}=\bar{n}_{ \pm}$. In this case, correlation functions are given by contributions of the form

$$
\frac{(\lambda / 2 k)^{n_{+}+n_{-}}}{n_{+} ! n_{-} !} \int \prod_{r=1}^{n_{+}} d^{2} w_{r} \prod_{l=1}^{n_{-}} d^{2} y_{l}\left\langle\prod_{\nu=1}^{N} \Phi_{j_{n}, m_{\nu}}^{s_{\nu}}\left(z_{\nu}\right) \prod_{r=1}^{n_{+}} \mathcal{S}_{++}\left(w_{r}\right) \prod_{l=1}^{n_{-}} \mathcal{S}_{--}\left(y_{l}\right)\right\rangle_{\text {free }}
$$

where the subscript "free" refers to the fact that this correlator is defined in the free field theory. The amount of screening insertions $n_{ \pm}$in (A.8) is given by (A.5) and (A.6) . Correlators similar to (A.8) but with a different amount of screening insertions $\prod_{r=1}^{n_{+}-n} \mathcal{S}_{++}\left(w_{r}\right)$ $\prod_{l=1}^{n_{-}-n} \mathcal{S}_{--}\left(y_{l}\right) \prod_{t=1}^{n} \mathcal{S}_{-+}\left(\hat{w}_{t}\right) \prod_{s=1}^{n} \mathcal{S}_{+-}\left(\hat{y}_{s}\right)$ also contribute. All the contributions are gathered with an appropriate prescription to integrate the screening operators in the worldsheet.

The inclusion of screening operators (A.2)-(A.3) in (A.8) can be also thought of as coming from the interaction terms in the action $S^{\mathrm{WZNW}}(g)$. In this picture, conditions (A.5)-(A.7) emerge from the integration over the zero-modes of the fields. The scale $\lambda$ is easily introduced by shifting the zero mode of $\phi$ as $\phi(z) \rightarrow \phi(z)+b^{-1} \log (\lambda)$. The parameter $\lambda$ allows to keep track of the KPZ scaling of correlation functions [45].

Correlation functions (A.8) can be computed by using free field propagators (2.4),

$$
\begin{aligned}
\left\langle\prod_{\nu=1}^{N} \Phi_{j_{n}, m_{\nu}}^{s_{\nu}}\left(z_{\nu}\right)\right\rangle= & \prod_{\nu=1}^{N} N_{j_{\nu}, m_{\nu}}^{s_{\nu}} \frac{(\lambda / 2 k)^{n_{+}+n_{-}}}{n_{+} ! n_{-} !} \int \prod_{r=1}^{n_{+}} d^{2} w_{r} \prod_{l=1}^{n_{-}} d^{2} y_{l} \\
& \times\left\langle\prod_{\nu=1}^{N} e^{i s_{\nu} Y\left(z_{\nu}\right)} \prod_{r=1}^{n_{+}} e^{-i Y\left(w_{r}\right)} \prod_{l=1}^{n_{-}} e^{i Y\left(y_{l}\right)}\right\rangle_{\text {free }} \times \\
& \times\left\langle\prod_{\nu=1}^{N} e^{b\left(2 j_{\nu}+1\right) \phi\left(z_{\nu}\right)} \prod_{r=1}^{n_{+}} e^{b \phi\left(w_{r}\right)} \prod_{l=1}^{n_{-}} e^{b \phi\left(y_{l}\right)}\right\rangle_{\text {free }} \times \\
& \times\left\langle\prod_{\nu=1}^{N} \gamma_{\left(z_{\nu}\right)}^{m_{\nu}-j_{\nu}-\left(s_{\nu}+1\right) / 2} \prod_{l=1}^{n_{-}} \beta_{\left(y_{l}\right)}\right\rangle_{\text {free }} \times \text { h.c. }
\end{aligned}
$$

where, again, $N_{j, m}^{s}$ is the normalization of the vertex. The standard normalization $N_{j, m}^{s}=$ $\frac{\Gamma(-j+1 / 2-s / 2+m)}{\Gamma(j+1 / 2+\bar{s} / 2+\bar{m})}$ yields

$$
\left\langle\Phi_{j, m}^{s}\left(z_{1}\right) \Phi_{-j-1 / 2,-m}^{1-s}\left(z_{2}\right)\right\rangle=\left|z_{1}-z_{2}\right|^{-4 \Delta}
$$

By expanding this expression, after Wick contracting all the contributions, it takes the form

$$
\begin{gathered}
\left\langle\prod_{\nu=1}^{N} \Phi_{j_{n}, m_{\nu}}^{s_{\nu}}\left(z_{\nu}\right)\right\rangle=\frac{1}{n_{+} ! n_{-} !}\left(\frac{\lambda}{2 k}\right)^{\left.-2\left(j_{1}+\ldots j_{N}\right)+1-N\right)} \prod_{\nu=1}^{N} N_{j_{\nu}, m_{\nu}}^{s_{\nu}} \times \\
\times \prod_{\mu<\nu}^{N}\left(z_{\mu}-z_{\nu}\right)^{s_{\mu} s_{\nu}-b^{2}\left(2 j_{\mu}+1\right)\left(2 j_{\nu}+1\right)} \int \prod_{r=1}^{n_{+}} d^{2} w_{r} \prod_{l=1}^{n_{-}} d^{2} y_{l} \times \\
\times \prod_{l=1}^{n_{-}} \prod_{r=1}^{n_{+}}\left(w_{r}-y_{l}\right)^{-1-b^{2}} \prod_{l<l^{\prime}}^{n_{-}}\left(y_{l}-y_{l^{\prime}}\right)^{1-b^{2}} \prod_{r<r^{\prime}}^{n_{+}}\left(w_{r}-w_{r^{\prime}}\right)^{1-b^{2}} \times \\
\times \prod_{\nu=1}^{N} \prod_{r=1}^{n_{+}}\left(z_{\nu}-w_{r}\right)^{-s_{\nu}-b^{2}\left(2 j_{\nu}+1\right)} \prod_{\nu=1}^{N} \prod_{l=1}^{n_{-}}\left(z_{\nu}-y_{l}\right)^{s_{\nu}-b^{2}\left(2 j_{\nu}+1\right)} \times
\end{gathered}
$$




$$
\times\left\langle\prod_{\nu=1}^{N} \gamma_{\left(z_{\nu}\right)}^{m_{\nu}-j_{\nu}-\left(s_{\nu}+1\right) / 2} \prod_{l=1}^{n_{-}} \beta_{\left(y_{l}\right)}\right\rangle_{\text {free }} \times h . c .
$$

In addition, we may resort to projective invariance to fix three points at $z_{1}=0, z_{2}=1$, and $z_{N}=\infty$.

The correlator of the $(\beta, \gamma)$ ghost fields in (A.10) yields a rather complicated expression in general. Nevertheless, it simplifies substantially in some particular cases. For instance, in the case of two insertions it reads [46, 47]

$$
\begin{gathered}
\left\langle\gamma_{\left(z_{1}=0\right)}^{m_{1}-j_{1}-\left(s_{1}+1\right) / 2} \gamma_{\left(z_{2}=1\right)}^{m_{2}-j_{2}-\left(s_{2}+1\right) / 2} \prod_{l=1}^{n_{-}} \beta_{\left(y_{l}\right)}\right\rangle_{\text {free }} \times \text { h.c. }=\prod_{l=1}^{n_{-}}\left|y_{l}\right|^{-2}\left|1-y_{l}\right|^{-2} \times \\
\times(-1)^{n_{-}} \frac{\Gamma\left(1 / 2-j_{1}-s_{1} / 2+m_{1}\right)}{\Gamma\left(1 / 2+j_{1}+s_{1} / 2-m_{1}\right)} \frac{\Gamma\left(1 / 2-j_{2}-s_{2} / 2+m_{2}\right)}{\Gamma\left(1 / 2+j_{2}+s_{2} / 2-m_{2}\right)} .
\end{gathered}
$$

World-sheet integral (A.10) can in principle be computed by using generalized Selberg integral formulas of the type worked out in [48, 49, 50]. To do this one has to give a precise prescription for contour integration. We will not address the details of such prescription in this appendix.

Integral representation (A.8) gathers the residues associated to the $N$-point correlation functions of the $\operatorname{OSP}(1 \mid 2)$ WZNW model, and after analytic continuation in $n_{ \pm}$and $j_{i}$ the exact form of the correlation functions are obtained. The exact expressions for twoand three-point correlation functions in the OSP(1|2) WZNW model were found in [21].

Representation (A.8) gives important information about the correlators. For instance, the KPZ scaling properties can be read from this expression. Correlators (A.10) scale as $\sim(\lambda / 2 k)^{n_{+}+n_{-}}$, where, according to (A.6) $), n_{+}+n_{-}=1-2\left(j_{1}+j_{2}+\ldots j_{N}\right)-N$. In particular, for the two-point function, where $N=2$ and $j_{1}=j_{2}=j$, we obtain $\sim(\lambda / 2 k)^{-4 j-1}$. So, let us compare this with the scaling properties of the exact exact solution of the OPS(1|2) WZNW model found in [21]. First, let us notice that in comparing the conventions of [21] with ours here we have to redefine $j$ as follows $j \rightarrow j+1 / 2$. Thus, the KPZ scaling is $\sim(\lambda / 2 k)^{-4 j-3}$ which precisely agrees with the result in [21]. Actually, it is not hard to see that if one introduces the scale $\lambda$ in the formulas of [21] then eq. (4.7) therein scales like

$$
\sim\left(\frac{2 k b^{2}}{i \lambda \gamma\left(\frac{b^{2}+1}{2}\right)}\right)^{4 j+3}
$$

Analogously, for the three-point functions one finds $\sim(\lambda / 2 k)^{-2\left(j_{1}+j_{2}+j_{3}\right)-5}$ which also coincides with the scaling of eq. (4.21) of [21].

One can also see that in the coincidence limit $\lim _{z_{1} \rightarrow z_{2}} \Phi_{j_{1} m_{1}}^{s_{1}}\left(z_{1}\right) \Phi_{j_{2} m_{2}}^{s_{2}}\left(z_{2}\right)$, where two of the vertices hit each other, the pole condition that appears at $z_{1}=z_{2}$ can be interpreted as a mass-shell condition $L_{0}-1=-2 b^{2} j(j+1 / 2)+s(s-1) / 2+l=0$ of a level- $l$ excited intermediate state carrying momenta $j=j_{1}+j_{2}-1+\left(N+n_{+}^{\prime}+n_{-}^{\prime}\right) / 2$ and $s=s_{1}+s_{2}+n_{+}^{\prime}-n_{-}^{\prime}$, where $n_{ \pm}^{\prime} \leq n_{ \pm}$are the amount of screening operators of the 
type $S_{ \pm}$whose inserting points also tend to $z_{2}$ together with $z_{1}$. In this limit, the $N$ point function factorizes in the product of one three-point function times one $N-2$-point function.

\section{References}

[1] J. M. Maldacena, "The large $N$ limit of superconformal field theories and supergravity," Adv. Theor. Math. Phys. 2 (1998) 231 [Int. J. Theor. Phys. 38 (1999) 1113] arXiv:hep-th/9711200;

[2] R. R. Metsaev and A. A. Tseytlin, "Type IIB superstring action in $A d S_{5} \times S^{5}$ background," Nucl. Phys. B 533, 109 (1998) arXiv:hep-th/9805028.

[3] N. Berkovits, C. Vafa and E. Witten, "Conformal field theory of AdS background with Ramond-Ramond flux," JHEP 9903, 018 (1999) arXiv:hep-th/9902098.

[4] T. Takayanagi and N. Toumbas, "A matrix model dual of type 0B string theory in two dimensions," JHEP 0307 (2003) 064 [arXiv:hep-th/0307083].

[5] M. R. Douglas, I. R. Klebanov, D. Kutasov, J. M. Maldacena, E. J. Martinec and N. Seiberg, "A new hat for the $c=1$ matrix model," arXiv:hep-th/0307195.

[6] T. Takayanagi, "Matrix model and time-like linear dilaton matter," JHEP 0412 (2004) 071 arXiv:hep-th/0411019.

[7] T. Takayanagi, "c $<1$ string from two dimensional black holes," JHEP 0507, 050 (2005) arXiv:hep-th/0503237.

[8] T. Okuda and T. Takayanagi, "Ghost D-branes," JHEP 0603 (2006) 062 arXiv:hep-th/0601024.

[9] N. Berkovits, "A new limit of the $A d S_{5} \times S^{5}$ sigma model," JHEP 0708, 011 (2007) arXiv:hep-th/0703282.

[10] N. Berkovits and C. Vafa, "Towards a worldsheet derivation of the Maldacena conjecture," JHEP 0803, 031 (2008) [AIP Conf. Proc. 1031, 21 (2008)] arXiv:0711.1799 [hep-th]].

[11] G. Bonelli and H. Safaai, "On gauge/string correspondence and mirror symmetry," JHEP 0806, 050 (2008) [arXiv:0804.2629 [hep-th]].

[12] N. Berkovits, "Perturbative super-Yang-Mills from the topological $A d S_{5} \times S^{5}$ sigma model," JHEP 0809, 088 (2008) arXiv:0806.1960 [hep-th]].

[13] G. Bonelli, P. A. Grassi and H. Safaai, "Exploring pure spinor string theory on $A d S_{4} \times \mathbb{C P}^{3}, "$ JHEP 0810, 085 (2008) arXiv:0808.1051 [hep-th]].

[14] S. Mukhi and C. Vafa, "Two-dimensional black hole as a topological coset model of $c=1$ string theory," Nucl. Phys. B 407, 667 (1993) arXiv:hep-th/9301083.

[15] M. Bershadsky and H. Ooguri, "Hidden $\operatorname{OSP}(N \mid 2)$ symmetries in superconformal field theories," Phys. Lett. B 229, 374 (1989). 
[16] M. Bershadsky and H. Ooguri, "Hidden SL(n) symmetry in conformal field theories," Comm. Math. Phys. 126 (1989) 49.

[17] Y. Kazama and H. Suzuki, "New $\mathcal{N}=2$ superconformal field theories and superstring compactification," Nucl. Phys. B 321, 232 (1989).

[18] Y. Kazama and H. Suzuki, "Characterization of $\mathcal{N}=2$ superconformal models generated by coset space method," Phys. Lett. B 216, 112 (1989).

[19] T. Creutzig, P. B. Ronne and V. Schomerus, " $\mathcal{N}=2$ superconformal symmetry in super coset models," arXiv:0907.3902 [hep-th].

[20] T. Creutzig, "Branes in supergroups," DESY-THESIS-2009-018.

[21] Y. Hikida and V. Schomerus, "Structure constants of the OSP(1|2) WZNW model," JHEP 0712, 100 (2007) arXiv:0711.0338 [hep-th]].

[22] S. Ribault and J. Teschner, " $H_{3}^{+}$WZNW correlators from Liouville theory," JHEP 0506, 014 (2005) arXiv:hep-th/0502048.

[23] Y. Hikida and V. Schomerus, " $H_{3}^{+}$WZNW model from Liouville field theory," JHEP 0710, 064 (2007) arXiv:0706.1030 [hep-th]].

[24] M. Wakimoto, "Fock representations of the affine lie algebra $A_{1}(1)$," Commun. Math. Phys. 104 (1986) 605.

[25] I. P. Ennes, A. V. Ramallo and J. M. Sanchez de Santos, "On the free field realization of the $\operatorname{osp}(1 \mid 2)$ current algebra," Phys. Lett. B 389, 485 (1996) arXiv:hep-th/9606180.

[26] I. P. Ennes, A. V. Ramallo and J. M. Sanchez de Santos, "Structure constants for the osp(1|2) current algebra," Nucl. Phys. B 491, 574 (1997) arXiv:hep-th/9610224.

[27] A. LeClair, "The gl(1|1) super-current algebra: the role of twist and logarithmic fields," arXiv:0710.2906 [hep-th].

[28] T. Creutzig and P. B. Ronne, "The GL(1|1)-symplectic fermion correspondence," Nucl. Phys. B 815, 95 (2009) [arXiv:0812.2835 [hep-th]].

[29] V. S. Dotsenko, "The free field representation of the SU(2) conformal field theory," Nucl. Phys. B 338 (1990) 747.

[30] V. S. Dotsenko, "Solving the SU(2) conformal field theory with the Wakimoto free field representation," Nucl. Phys. B 358, 547 (1991).

[31] M. Bershadsky and D. Kutasov, "Comment on gauged WZW theory," Phys. Lett. B 266, 345 (1991).

[32] R. Dijkgraaf, H. L. Verlinde and E. P. Verlinde, "String propagation in a black hole geometry," Nucl. Phys. B 371, 269 (1992).

[33] J. M. Maldacena and H. Ooguri, "Strings in $A d S_{3}$ and SL(2,R) WZW model. I," J. Math. Phys. 42, 2929 (2001) arXiv:hep-th/0001053.

[34] T. Eguchi and S. K. Yang, " $\mathcal{N}=2$ superconformal models as topological field theories," Mod. Phys. Lett. A 5 (1990) 1693. 
[35] E. Witten, "Mirror manifolds and topological field theory," arXiv:hep-th/9112056.

[36] S. Nakamura and V. Niarchos, "Notes on the S-matrix of bosonic and topological non-critical strings," JHEP 0510, 025 (2005) arXiv:hep-th/0507252].

[37] S. Ribault, "Knizhnik-Zamolodchikov equations and spectral flow in $A d S_{3}$ string theory," JHEP 0509, 045 (2005) arXiv:hep-th/0507114.

[38] G. Giribet, "The string theory on $A d S_{3}$ as a marginal deformation of a linear dilaton background," Nucl. Phys. B 737, 209 (2006) arXiv:hep-th/0511252].

[39] Y. Hikida and V. Schomerus, "The FZZ-duality conjecture - A proof," arXiv:0805.3931 [hep-th].

[40] R. Dijkgraaf, H. L. Verlinde and E. P. Verlinde, "Topological strings in $d<1$," Nucl. Phys. B 352, 59 (1991).

[41] M. Bershadsky, S. Cecotti, H. Ooguri and C. Vafa, "Kodaira-Spencer theory of gravity and exact results for quantum string amplitudes," Commun. Math. Phys. 165 (1994) 311 [arXiv:hep-th/9309140].

[42] V. Fateev, A.B. Zamolodchikov and Al. Zamolodchikov, unpublished note.

[43] K. Hori and A. Kapustin, "Duality of the fermionic $2 \mathrm{~d}$ black hole and $\mathcal{N}=2$ Liouville theory as mirror symmetry," JHEP 0108 (2001) 045 [arXiv:hep-th/0104202].

[44] V. Kazakov, I. K. Kostov and D. Kutasov, "A matrix model for the two-dimensional black hole," Nucl. Phys. B 622, 141 (2002) arXiv:hep-th/0101011].

[45] V. G. Knizhnik, A. M. Polyakov and A. B. Zamolodchikov, "Fractal structure of 2d-quantum gravity," Mod. Phys. Lett. A 3, 819 (1988).

[46] K. Becker and M. Becker, "Interactions in the SL $(2, \mathbb{R})$ / U(1) black hole background," Nucl. Phys. B 418, 206 (1994) |arXiv:hep-th/9310046|.

[47] G. Giribet and C. A. Nunez, "Correlators in $A d S_{3}$ string theory," JHEP 0106, 010 (2001) arXiv:hep-th/0105200|.

[48] V. S. Dotsenko and V. A. Fateev, "Four point correlation functions and the operator algebra in the two-dimensional conformal invariant theories with the central charge $c<1$," Nucl. Phys. B 251, 691 (1985).

[49] T. Fukuda and K. Hosomichi, "Three-point functions in sine-Liouville theory," JHEP 0109, 003 (2001) [arXiv:hep-th/0105217].

[50] T. Fukuda and K. Hosomichi, "Super Liouville theory with boundary," Nucl. Phys. B 635, 215 (2002) arXiv:hep-th/0202032. 